\title{
Influence of a family history of breast and/or ovarian cancer on breast cancer outcomes
}

\author{
A-YONG CAO, MIN HE, GEN-HONG DI, JIONG WU, JIN-SONG LU, \\ GUANG-YU LIU, ZHEN-ZHOU SHEN and ZHI-MING SHAO
}

\begin{abstract}
Breast Cancer Institute, Cancer Hospital/Cancer Institute, Department of Oncology, Shanghai Medical College, Institutes of Biomedical Science, Fudan University, Shanghai, P.R. China
\end{abstract}

Received March 21, 2011; Accepted May 16, 2011

DOI: $10.3892 /$ etm.2011.275

\begin{abstract}
Various published studies have been inconclusive in attempting to relate a family history of breast and/or ovarian cancer (BOC) to the survival of breast cancer patients. The aim of the study was to investigate the association of a family history of BOC with tumor characteristics, treatment response and the difference between the prognosis of familial breast cancer (FBC) patients and sporadic breast cancer (SBC) patients. Data on 348 operable FBC patients and 345 SBC patients were retrospectively analyzed. The overall survival (OS) and recurrence/metastasis-free survival (RFS) were compared for both groups. FBC cases were diagnosed at a relatively younger age $(51.1 \pm 10.4$ vs. $53.7 \pm 11.0$ years, $\mathrm{P}=0.054)$ and presented a lower $\mathrm{T}$ stage $(\mathrm{P}=0.000)$ than the $\mathrm{SBC}$ cases. Patients with a family history of BOC had a significantly greater risk of recurrence/metastasis $(\mathrm{P}=0.04)$ and a non-significantly increased risk of death $(\mathrm{P}=0.06)$ compared to the SBC patients. In a multivariate analysis, family history of BOC was an independent predictive factor for both recurrence/metastasis rate $(\mathrm{P}=0.01, \mathrm{HR}=0.012,95 \% \mathrm{CI} 0.02-0.57)$ and mortality $(\mathrm{P}=0.044, \mathrm{HR}=0.43,95 \% \mathrm{CI} 0.19-0.98)$ in the hormone receptor-positive population. Our results found that women diagnosed with FBC had an early onset of disease in the population studied, and the poor outcome of patients with a family history of BOC associated with survival was restricted to the hormone receptor-positive population.
\end{abstract}

\section{Introduction}

Breast cancer is one of the leading causes of cancer-related morbidity and mortality in women worldwide, and its inci-

Correspondence to: Dr Zhi-Ming Shao, Breast Cancer Institute, Cancer Hospital/Cancer Institute, Department of Oncology, Shanghai Medical College, Fudan University, 270 Dong'an Road, Shanghai 200032, P.R. China

E-mail: zhimingshao@yahoo.com

Key words: family history, breast and/or ovarian cancer, breast cancer outcomes dence has been rapidly increasing in China (1). Most breast cancers are sporadic and develop in response to the cumulative effect of environmental risk factors and genetic susceptibility of individuals (2). A family history of breast or ovarian cancer (BOC) and early age at diagnosis are considered potential indicators of an underlying genetic predisposition for breast cancer (BC), and approximately $20 \%$ of breast cancers are associated with a clear family history of the disease (3). The association of a family history with presentation and outcome of breast cancer has been investigated in several studies, but the results are conflicting and few studies were populationbased using highly reliable family history and clinical data.

Several of these studies have reported that tumors in women with a family history of BC are more likely to be smaller, poorly differentiated, oestrogen receptor (ER)-negative/progesterone receptor (PR)-negative, and often exhibit lymphovasular invasion, which is associated with a less favorable prognosis $(4,5)$. However, other studies have yielded mixed results. They did not find evidence that a family history of $\mathrm{BC}$ is associated with tumor size, nodal status, hormone receptor status or grade (6). Therefore, there is no consensus regarding the association of clinicopathological characteristics and outcomes of familial breast cancer (FBC), and whether this association differs from sporadic breast cancer (SBC) in regards to prognostic factors.

In the present study, the association of the prognosis of FBC with clinical and pathological factors, treatment, recurrence/metastasis-free survival (RFS) and overall survival (OS) was retrospectively investigated in a Chinese population.

\section{Patients and methods}

Patients and follow-up. A total of 693 patients, who were diagnosed with $\mathrm{BC}$ through histopathology and treated at the Department of Breast Surgery at the Cancer Hospital/Institute, Fudan University (Shanghai, China) during the period January 1, 1994 to December 31, 2004 were enrolled in the present study. These patients were divided into an FBC group (348 cases) and an SBC group (345 cases), and all cases were females without distant metastasis at initial diagnosis, and with infiltrative carcinoma according to the inclusion criteria. In addition, all FBC cases were from a family with $\geq 2$ patients with BOC among the first-degree relatives, including the 
proband, regardless of age. The family histories concerning three-generation pedigrees of the eligible cases were retrieved from the medical records and standard questionaires, ascertained by the families and/or the patients personally.

According to the numbers of FBC patients who were enrolled every year during the study period, similar numbers of SBC patients were randomly selected in the corresponding year, and a total of 345 SBC cases without a family history of BOC or other malignancies in three generations recruited from the Cancer Hospital of Fudan University were enrolled as controls. All patients were required to undergo a complete physical examination, bilateral mammography, chest radioscopy, ECG, ultrasonography of the breasts, axillary fossa, cervical regions, abdomen and pelvis, and routine blood and biochemical tests before surgery and accompanying adjuvant therapy, according to surgical standards. All patients who presented a risk of relapse received adjuvant chemotherapy of different regimens for 4-6 cycles followed by local radiotherapy (if required) and/or hormone therapy (if required) according to the standard therapy at the time of surgery. This research project was approved by the Scientific and Ethics Committee of the Cancer Hospital of Fudan University.

Follow-up data were collected annually from medical records for breast cancer recurrence, new primary cancers and death. Personal contact with the patient, through routine correspondence or telephone visits, was used for follow-up. The patient follow-up was carried out at the Cancer Hospital of Fudan University every 3 months during the first 2 years, every 6 months during the next 2 years and once a year thereafter.

Methods for biological characteristics. Immunohistochemical status of each postoperative paraffin-embedded tumor sample was defined through immunohistochemical staining, including ER, PR and HER2/neu. All of the primary monoclonal antibodies were purchased from Dako, Hamburg, Germany. The detailed staining procedures were strictly followed according to the instructions provided with the reagents. Negative controls were obtained by incubation of parallel slides omitting the primary antibodies. Sections known to be stained positively in each run served as positive controls. The percentage and intensity score of the stained tumor cells (ER, PR, HER2/neu, P53, cathepsin-D and PCNA) were determined by at least two independent pathologists. The percentage was interpreted as follows: 0 , no staining; 1 , $\leq 25 \%$ positively stained cells; $2,25-50 \%$ positively stained cells; 3, 50-75\% positively stained cells; and 4, >75\% positively stained cells. Regarding the intensity, the score was as follows: 0 , negative; 1 , weakly positive; 2 , moderately positive; 3 , strongly positive. The percentage and intensity scores were combined to produce a final score. For all markers except HER2/neu, a score of 0 was defined as negative and 1-12 as positive, while merely scores of 9-12 for strong membranous staining (Dako score $3+$ ) were defined as positive.

Statistical analysis. The association of clinicopathological factors was evaluated using Pearson's Chi-square or Fisher's exact tests. The primary clinical outcomes for this study were RFS and OS. OS was defined as the time from initial diagnosis of primary $\mathrm{BC}$ to death from any cause, and RFS was defined as the time from initial diagnosis to local recurrence or metastasis. Survival time was calculated from the date of surgery to these endpoints, censoring at the date of last contact and non-breast primaries. The 5-year survival rate was calculated by the Life Tables method, and survival curves were obtained by the Kaplan-Meier method. The log-rank test was used to determine the statistical significance in comparative survival for a variety of patient and tumor characteristics. All the statistically significant variables observed in the univariate analysis were investigated by means of multivariate analysis using the Cox proportional hazards model. All P-values $<0.05$ were considered statistically significant. All P-values were two-sided. SPSS 15.0 software package (SPSS Inc.) was used for statistical analysis.

\section{Results}

Characteristics of the patients. The FBC group was comprised of 348 cases who were between 26 and 87 years of age (mean 49.3). The SBC group was comprised of 345 cases who were between 27 and 87 years of age (mean 56.3). The mean age of the FBC cases was significantly younger compared to the SBC cases $(51.1 \pm 10.4$ vs. $53.7 \pm 11.0$ years), likely reflecting that the patients with a genetic risk exhibited an early-onset of disease. The age distributions and characteristics of the two groups are listed in Table I. The FBC group exhibited a relatively lower T stage $(\mathrm{P}=0.00)$, while the FBC cases did not differ from the SBC group in terms of the lymph node-positive rate and grade of tumor differentiation, and there was no significant differences in ER or PR status when the unknowns were excluded between the two groups. Regarding hormone receptor (ER or PR) status, the SBC group had a relatively higher hormone receptor-positive rate $(\mathrm{P}=0.05)$. As for HER2/neu, cathepsin$\mathrm{D}$ and PCNA, the two groups had a similar amplification status $(\mathrm{P}=0.0 .47$ for HER2/neu, $\mathrm{P}=0.33$ for cathepsin-D and $\mathrm{P}=0.14$ for $\mathrm{PCNA}$ ). With regard to the method of adjuvant treatment, the SBC individuals were more likely to receive adjuvant chemotherapy or hormone therapy compared to the $\mathrm{FBC}$ cases $(\mathrm{P}=0.000$ and $\mathrm{P}=0.000$, respectively), while a similar radiotherapy proportion $(\mathrm{P}=0.168)$ was noted. In addition, no statistically significant differences in terms of surgery were noted between the two groups.

The median follow-up was 55.7 months (range 6-120); 52.3 months for the FBC group vs. 61.1 months for the SBC group. A total of 46 patients were lost during the follow-up period.

Univariate survival analysis. The 5-year OS was 94 vs. 98\%, and the 5-year RFS was 85 vs. $87 \%$ in the FBC and SBC groups, respectively. Upon comparison between the two groups, different outcomes were revealed.

The patients with a family history of BOC had a significantly higher risk of recurrence/metastasis $(\mathrm{P}=0.04)$ and a non-significantly increased risk of death $(\mathrm{P}=0.06)$ (Fig. 1A and $\mathrm{B}$ ) regardless of age and TNM stage. Family history was one of the major predictors of worse survival in the elder ( $>40$ years of age) group, the higher TNM stage group and the hormone receptor-positive group. After adjustment for age, a borderline significant difference was observed in the elder subgroup ( $>40$ years) $(\mathrm{P}=0.06$ for $\mathrm{OS}$ and $\mathrm{P}=0.12$ for 
Table I. Clinicopathological characteristics of the FBC and SBC patients.

\begin{tabular}{|c|c|c|c|}
\hline \multirow[t]{2}{*}{ Characteristics } & $\begin{array}{c}\text { FBC } \\
n=348\end{array}$ & $\begin{array}{c}\text { SBC } \\
n=345\end{array}$ & P-value \\
\hline & No. $(\%)$ & No. $(\%)$ & \\
\hline
\end{tabular}

Age (years)
$\leq 40$
$>40$

Surgery

Mastectomy

BCS

Tumour size $(\mathrm{cm})$

$\mathrm{T} \leq 2$
$2<\mathrm{T} \leq 5$
$\mathrm{~T}>5$
$\mathrm{Tx}$

Lymph node status

0

1-3

4-10

$>10$

Unknown

Grade of differentiation

$$
\text { I }
$$

II

III

Unknown

ER status

Negative

Positive

Unknown

PR status

Negative

Positive

Unknown

HER2/neu status

Negative

Positive

Unknown

Chemotherapy

No
MTX-containing
Anthrocyclin-containing
Taxane-containing
Others
Unknown
Radiation therapy
No
Yes
Unknown
Hormone therapy
No
Yes

Unknown

$\begin{array}{rr}54(18.4) & 36(10.4) \\ 294(81.6) & 309(89.6) \\ & \\ 310(89.1) & 314(91.0) \\ 38(10.9) & 31 \quad(9.0) \\ & \\ 117(33.6) & 66(19.1) \\ 180(51.8) & 214(62.0) \\ 14(4.0) & 23(6.7) \\ 37(10.6) & 42(12.2)\end{array}$

0.054

0.447

0.000

$180(51.8) \quad 174(50.4)$

$74(21.2) \quad 52(15.1)$

45 (12.9) $26 \quad(7.5)$

$\begin{array}{llll}10 & (2.9) \quad 11 \quad(3.2)\end{array}$

39 (11.2) $82(23.8)$

$5 \quad(1.4) \quad 8 \quad(2.3)$

$142(40.8) \quad 175(50.7)$

$\begin{array}{llll}33 & (9.5) \quad 32 & (9.3)\end{array}$

$168(48.3) \quad 130(37.7)$

$94(27.0) \quad 121(35.1)$

$170(48.9) \quad 188(54.5)$

$84(24.1) \quad 36(10.4)$

110 (31.6) $152(44.1)$

147 (42.2) $154(44.6)$

91 (26.2) $39(11.3)$

$200(57.5) \quad 232(67.2)$

$51(14.6) \quad 69(20.0)$

97 (27.9) $\quad 44(12.8)$

$63(18.1) \quad 32 \quad(9.3)$

$93(26.7) \quad 56(16.2)$

$160(46.0) \quad 219(63.5)$

$\begin{array}{llll}6 & (1.7) \quad 3 & (0.9)\end{array}$

$12 \quad(3.5) \quad 15 \quad(4.3)$

$14 \quad(4.0) \quad 20 \quad(5.8)$

$314(90.2) \quad 291(84.3)$

$\begin{array}{llll}24 & (6.9) \quad 33 & (9.6)\end{array}$

$10(2.9) \quad 21 \quad(6.1)$

$258(74.2) \quad 142(41.2)$

85 (24.4) $198(57.4)$

$5 \quad(1.4) \quad 5 \quad(1.4)$

0.144

0.592

0.168
Table I. Continued.

\begin{tabular}{|c|c|c|}
\hline \multirow[t]{2}{*}{ Characteristics } & $\begin{array}{c}\text { FBC } \\
n=348\end{array}$ & $\begin{array}{c}\mathrm{SBC} \\
\mathrm{n}=345\end{array}$ \\
\hline & No. $(\%)$ & No. $(\%)$ \\
\hline
\end{tabular}

P53

$\begin{array}{lrr}\text { Negative } & 94(27.0) & 172(49.9) \\ \text { Positive } & 138(39.7) & 127(36.8) \\ \text { Unknown } & 116(33.3) & 46(13.3) \\ \text { Cathepsin-D } & & \\ \text { Negative } & 52(15.0) & 63(18.3) \\ \text { Positive } & 156(44.8) & 237(68.7) \\ \text { Unknown } & 140(40.2) & 45(13.0) \\ \text { PCNA } & & \\ \text { Negative } & 219(62.9) & 163(47.2) \\ \text { Positive } & 80(23.0) & 43(12.5) \\ \text { Unknown } & 49(14.1) & 139(40.3)\end{array}$

0.000

0.332

0.141

FBC, familial breast cancer; SBC, sporadic breast cancer; BCS, breast-conserving surgery.

RFS) (Fig. 1C and D). When TNM stage was included in the analysis, the FBC patients exhibited a significantly increased risk for RFS and decreased OS compared to the SBC cases in the higher TNM stage subgroup $(\mathrm{P}=0.09$ for $\mathrm{OS}$ and $\mathrm{P}=0.02$ for RFS) (Fig. 1E and F). In univariate analysis, there was also an increased risk of recurrence/metastasis for the FBC women when compared to the SBC cases in the subgroup with hormone receptor-positive tumors $(\mathrm{P}=0.01$ for $\mathrm{OS}$, and $\mathrm{P}=0.01$ for RFS) (Fig. 2A and B). Overexpression of HER2/neu is considered to be an unfavorable predictor for RFS or OS in

0.108 breast cancer patients, but the difference was more significant in the FBC patients with HER2/neu-negative status $(\mathrm{P}=0.04$ for OS and $\mathrm{P}=0.12$ for RFS) in the present analysis (Fig. $2 \mathrm{C}$ and D).

0.470 The response rate of systemic adjuvant therapy in the FBC patients was lower than that of the SBC cases, particularly for chemotherapy and hormone therapy. The familial BC women who received chemotherapy had a higher rate of death and recurrence/metastasis $(\mathrm{P}=0.03)$ compared to their counterparts (Fig. 3A and B). Regarding the patients who received hormone therapy, the difference was more significant $(\mathrm{P}=0.00$ for OS and $\mathrm{P}=0.01$ for RFS) (Fig. 3C and D). Additionally, when administered hormone therapy after chemotherapy, the FBC group experienced more recurrence/metastasis events, and the OS difference reached statistical significance (data not shown).

Multivariate survival analysis. Upon univariate survival analysis, a significantly increased risk for RFS and decreased OS was observed for the familial cases compared to the SBC group in the hormone receptor-positive population $(\mathrm{P}=0.01$ for OS and $\mathrm{P}=0.01$ for RFS) in contrast to the hormone receptornegative population ( $\mathrm{P}=0.82$ for $\mathrm{OS}$ and $\mathrm{P}=0.23$ for $\mathrm{RFS})$; therefore, multivariate survival analysis was further performed in the hormone receptor-positive population. Family history 

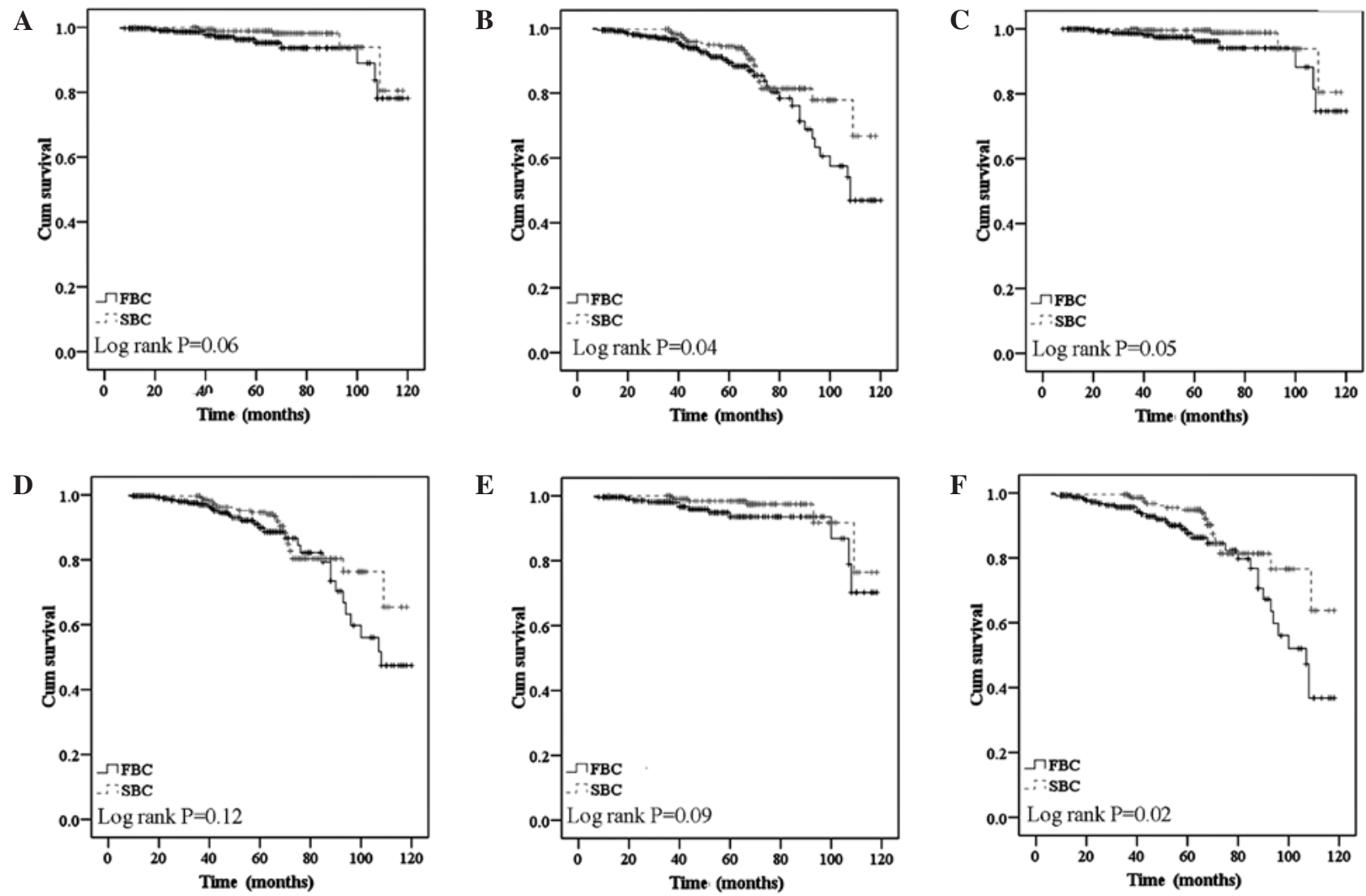

Figure 1. (A) Overall survival (OS) and (B) recurrence/metastasis-free survival (RFS) according to family history; (C) OS and (D) RFS in a population $\geq 40$ years of age; (E) OS and (F) RFS in a population with higher TNM stage. "Adjusted for tumor size ( $\leq 2 \mathrm{vs.}>2 \mathrm{~cm}$ ) and lymph node status (positive vs. negative).

A

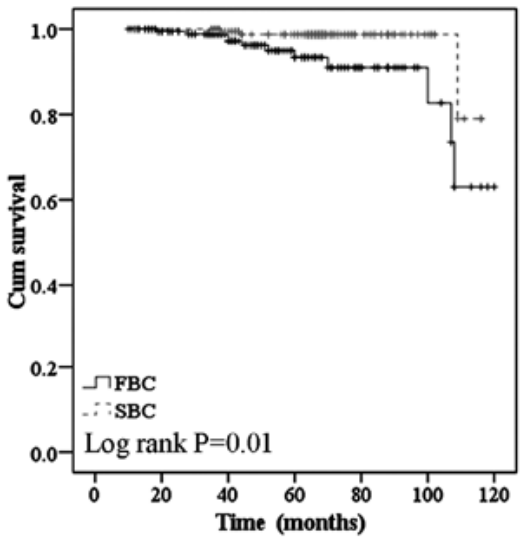

C

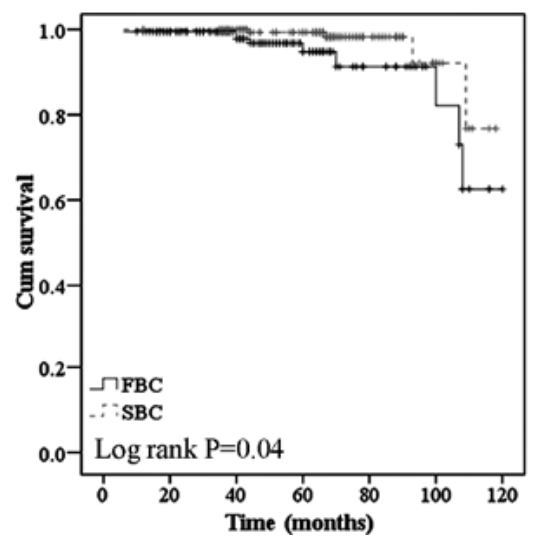

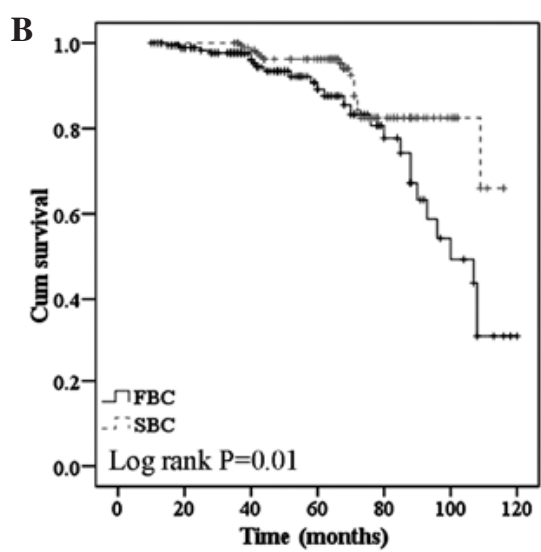

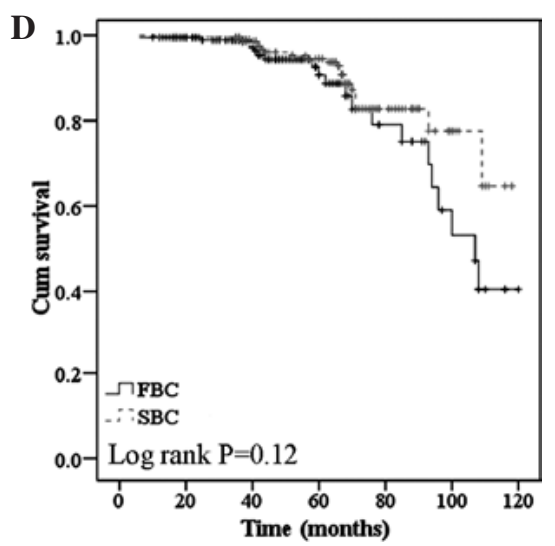

Figure 2. (A) Overall survival (OS) and (B) recurrence/metastasis-free survival (RFS) according to hormone receptor-positive status; (C) OS and (D) RFS in a human epidermal growth factor receptor 2-negative population. ${ }^{*}$ Adjusted for tumor size $(\leq 2 \mathrm{vs} .>2 \mathrm{~cm}$ ) and lymph node status (positive vs. negative). 

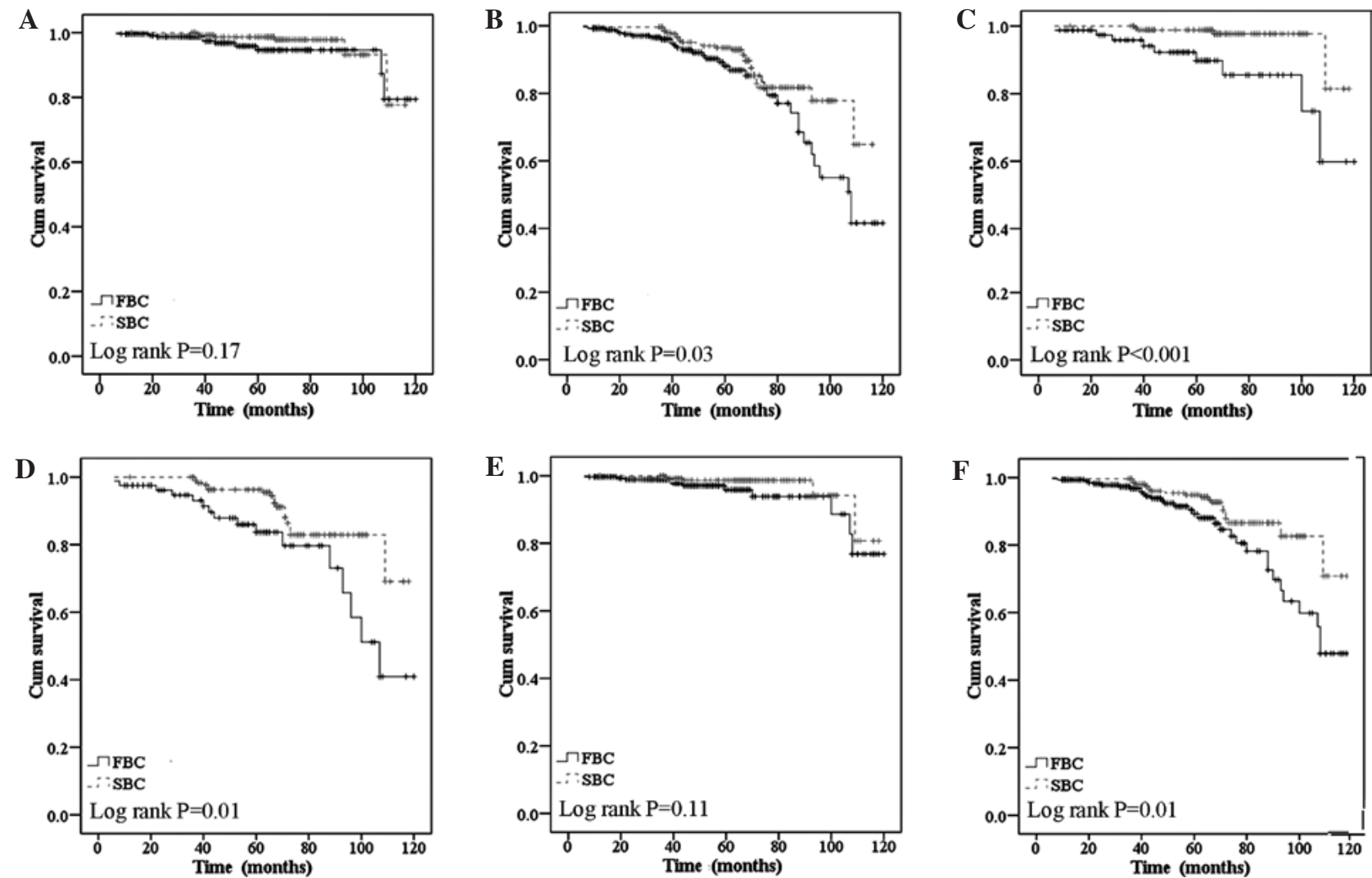

Figure 3. (A) Overall survival (OS) and (B) recurrence/metastasis-free survival (RFS) according to chemotherapy; (C) OS and (D) RFS in patients receiving hormone therapy; (E) OS and (F) RFS in patients receiving no radiotherapy. "Adjusted for tumor size ( $\leq 2 \mathrm{vs} .>2 \mathrm{~cm}$ ) and lymph node status (positive vs. negative).

Table II. Cox's proportional hazards regression model for the general hormone receptor-positive population.

\begin{tabular}{lc}
\hline Variables $\quad$ P-value $\quad$ HR $\begin{array}{c}95 \% \mathrm{CI} \text { for } \\
\text { hormone } \\
\text { receptor }\end{array}$
\end{tabular}

For general OS

$\begin{array}{llll}\text { Without family history } & 0.044 & 0.43 & 0.19-0.98 \\ \text { Age (years) } \geq 40 & 0.099 & 0.28 & 0.06-1.27 \\ \text { Higher TNM stage } & 0.023 & 5.31 & 0.60-8.88 \\ \text { HER2/neu negative } & 0.187 & 0.33 & 0.06-1.72 \\ \text { Chemotherapy } & 0.947 & 0.94 & 0.18-5.04 \\ \text { Radiotherapy } & 0.275 & 0.26 & 0.02-2.92 \\ \text { Hormone therapy } & 0.137 & 0.78 & 0.23-2.60 \\ \text { For general RFS } & & & \\ \text { Without family history } & 0.010 & 0.32 & 0.02-0.57 \\ \text { Age (years) } \geq 40 & 0.612 & 0.79 & 0.32-1.97 \\ \text { Higher TNM stage } & 0.040 & 1.81 & 0.75-4.39 \\ \text { HER2/neu-negative } & 0.737 & 0.87 & 0.39-1.94 \\ \text { Chemotherapy } & 0.874 & 1.11 & 0.32-3.85 \\ \text { Radiotherapy } & 0.190 & 1.05 & 0.42-2.65 \\ \text { Hormone therapy } & 0.299 & 0.67 & 0.32-1.42\end{array}$

OS, overall survival; RFS, recurrence/metastasis-free survival; HR, hazard ratio; $\mathrm{CI}$, confidence interval.

of BOC was identified to be an independent predictive factor for both the recurrence/metastasis rate $(\mathrm{P}=0.01, \mathrm{HR}=0.012$,
95\% CI 0.02-0.57) and mortality $(\mathrm{P}=0.044, \mathrm{HR}=0.43,95 \% \mathrm{CI}$ 0.19-0.98) (Table II).

Higher TNM stage $(\mathrm{P}=0.023, \mathrm{HR}=5.31,95 \%$ CI $0.60-8.88$ for OS and $\mathrm{P}=0.04, \mathrm{HR}=1.81,95 \%$ CI $0.75-4.39$ for RFS) indicated a poor prognosis for OS, no matter whether the patients had a family history of BOC $(\mathrm{P}=0.012, \mathrm{HR}=4.42,95 \% \mathrm{CI}$ 0.45-6.70 in the FBC group and $\mathrm{P}=0.03, \mathrm{HR}=3.33,95 \% \mathrm{CI}$ 1.39-4.79 in the SBC group; Table III), whereas HER2/neu amplification did not predict a worse survival $(\mathrm{P}=0.19$ for $\mathrm{OS}$ and $\mathrm{P}=0.74$ for $\mathrm{RFS}$ ).

Moreover, adjuvant therapy had a tendency to reduce the risk of recurrence/metastasis $(\mathrm{P}=0.060, \mathrm{HR}=0.69,95 \% \mathrm{CI}$ 0.4-1.02) in the SBC group, but not in the FBC group. Although there was similar rate $(\mathrm{P}=0.10)$ of receiving chemotherapy between the two groups in the hormone receptor-positive population, a borderline difference was still observed in the SBC individuals ( $\mathrm{P}=0.06)$, while in the $\mathrm{FBC}$ group the $\mathrm{P}$-value became 0.82 . A similar consequence was noted in the patients who received radiotherapy $(\mathrm{P}=0.07)$. Hormone therapy had a tendency to reduce the risk of recurrence/metastasis $(\mathrm{P}=0.229$, $\mathrm{HR}=0.67,95 \% \mathrm{CI} 0.32-1.42$ ) in the general hormone receptorpositive population, which was demonstrated in the SBC group $(\mathrm{P}=0.035, \mathrm{HR}=0.31,95 \% \mathrm{CI} 0.08-1.22)$, but not in the FBC group $(\mathrm{P}=0.93, \mathrm{HR}=1.04,95 \%$ CI 0.44-2.46).

In our cohort, HER2/neu overexpression did not demonstrate the opposite prognostic value in both groups $(\mathrm{P}=0.41$ in the FBC group and $\mathrm{P}=0.076$ in the SBC group; Table III).

As expected, the Cox's proportional hazard regression model for the FBC and SBC groups was consistent with the findings revealed in the univariate analysis. 
Table III. Cox's proportional hazards regression model for the FBC and SBC hormone receptor-positive groups.

\begin{tabular}{lccc}
\hline Variables & P-value & HR & $\begin{array}{c}95 \% \text { CI for } \\
\text { hormone } \\
\text { receptor }\end{array}$ \\
\hline For FBC & & & \\
Age (years) $\geq 40$ & 0.436 & 0.48 & $0.08-3.01$ \\
Higher TNM stage & 0.019 & 4.42 & $0.45-6.70$ \\
HER2/neu-negative & 0.408 & 0.49 & $0.09-2.67$ \\
Chemotherapy & 0.824 & 0.83 & $0.16-4.40$ \\
Radiotherapy & 0.440 & 0.38 & $0.03-4.51$ \\
Hormone therapy & 0.932 & 1.04 & $0.44-2.46$ \\
For SBC & & & \\
Age (years) $\geq 40$ & 0.561 & 0.52 & $0.06-4.72$ \\
Higher TNM stage & 0.032 & 3.33 & $1.39-4.79$ \\
HER2/neu-negative & 0.759 & 0.78 & $0.16-3.90$ \\
Chemotherapy & 0.059 & 0.10 & $0.01-1.09$ \\
Radiotherapy & 0.071 & 0.35 & $0.01-1.11$ \\
Hormone therapy & 0.035 & 0.31 & $0.08-1.22$ \\
\hline
\end{tabular}

FBC, familial breast cancer; SBC, sporadic breast cancer; HR, hazard ratio; $\mathrm{CI}$, confidence interval.

\section{Discussion}

Several risk factors for the etiology of breast cancer have also been correlated with the prognosis of breast cancer (7). As compared to SBCs, a positive family history of BOC appears to be a risk factor for the development of breast cancer, and in medical practice, a family history of cancers is vital information. Most familial cases are thought to be caused by an inherited genetic disorder (8). Although many studies have focused on germline mutations of breast cancer susceptibility genes which may contribute to FBCs, knowledge concerning the outcome of FBC remains to be elucidated.

Several studies have reported that individuals with a family history of BOC are more likely to present with smaller tumors and a higher grade of malignancy than those without a family history (9), while others studies have failed to find these associations $(10,11)$. Women with a family history of BOC in our study were characterized by early disease onset and relatively smaller tumor sizes. Two possible explanations may be i) that these women were affected by genetic factors and predisposed to an early-onset of breast cancer possibly attributable in part to more frequent BRCA1 or BRCA2 mutations in the familial women (8); and ii) that individuals with a familial history of BOC prefer to undergo early and regular routine screening of the breasts and present with a small tumor size at the initial diagnosis (12). Additionally, our results also showed that the familial tumors had a similar lymph node-positive rate, differentiation grade and hormone receptor expression status as the sporadic ones. There were no differences in clinical management when a family history of BOC was present. The two groups had a similar rate of receiving breast-conserving (BCS) therapy, and we did not observe more frequent recurrence/metastasis or death events in the BCS population (data not shown). This suggests that a family history of BOC did not have an impact on the treatment decisions.

The Chinese women with a family history of BOC were significantly younger of diagnosis, and appeared to have a worse RFS in contrast to their SBC counterparts, suggesting that a family history had a negative effect on RFS, but not OS. However, this prognostic impact of family history was more significant after stratification for age, TNM stage and different adjuvant therapies. In multivariate analysis using the Cox regression model with factors such as age, TNM stage, HER2/ neu status and adjuvant treatment methods, age $\geq 40$ years became a favorable factor in this model, with a relative risk ratio of 0.28 for OS and 0.78 for RFS, and no obvious effect of adjuvant chemotherapy or hormone therapy was detectable in the hormone receptor-positive population.

Breast cancer in young women often presents with more unfavourable characteristics and a lower response rate to systemic adjuvant therapy, which results in a poor prognosis. These observations have been validated by several large population-based studies (13-17). Upon univariate, adverse prognostic effects of family history appeared to be restricted to women with hormone-responsive breast cancer, the majority of whom received adjuvant chemotherapy. As the FBC women in the hormone receptor-positive population did not gain as much benefit from hormone therapy as their SBC counterparts, this poor outcome may have been due to the more young breast cancer cases and more inherent aggressive tumor phenotypes (such as HER2/neu overexpression) were present in the FBC group, which would probably lead to their resistance to tamoxifen treatment $(18,19)$.

Although we found that p53 had a significantly abnormal expression in the FBC group, the levels of p53 did not become an independent prognostic factor in the present study (data not shown). Finally, HER2/neu overexpression is believed to be an important factor in breast cancer survival prediction (20), but we failed to identify its effect in both groups. Considering that our findings were achieved in a limited sample of hormone receptor-positive tumors and the introduction of trastuzumab and similar targeted drugs against HER2/neu has dramatically altered the prognosis in patients with tumors expressing HER2/neu, the worse prognosis for patients with HER2-expressing tumors in our series during a period before the widespread use of trastuzumab should be taken into account.

In our retrospective study, we constructed three-generation cancer pedigrees and verified cases of BOC in relatives where possible, and then we focused on first-degree family history which has been shown to be valid and reproducible (21). Even though a detailed investigation in Chinese women was carried out, many more patients should be recruited in this cohort, and a longer follow-up time exceeding 10 years is required to improve the statistical power. In addition, the mutation frequency of BRCA1/BRCA2 in the Chinese FBC cases was reported to be more than $10 \%$ (1), and histopathological studies of BRCA-associated breast cancers have revealed features generally associated with a poor prognosis $(22,23)$. However we were unable to assess the BRCA1/BRCA2 mutation status in our cohort in view of that few FBC cases had undergone BRCA mutation testing; further studies should be carried out on BRCA1/BRCA2 mutation and prognosis of 
familial breast cancer patients in China as well as the targeted therapy related to this point.

We found that Chinese women with a family history of BOC had a significantly worse prognosis, but this should be further validated in larger datasets having greater statistical power to see whether a modest survival benefit is present in SBC cases. A prognostic effect of a family history identified in our analysis was not consistent with a number of other studies. Although it has never been shown that family history is related to shorter survival in a single population-based study (24), a significantly longer survival was demonstrated in two other studies $(4,25)$. However, the latter studies were restricted to a small sample of young pre-menopausal Caucasian women. In addition, there were differences in the analyzed ethnic groups, data quality and statistical power, even in the definition of family history.

Although various studies have reported their own observations, there is still no worldwide consensus with regard to the relationship of family history and RFS in breast cancer, and also the specific risk factors for familial women $(26,27)$. It is of interest to attempt to elucidate the possible differences in the genetic alterations in tumors of familial patients. Then, a comprehensive knowledge of the potential clinical importance of various aspects of genetic risk will help improve our understanding of the biological heterogeneity in breast cancer, and thus may lead to better clinical management of breast cancer patients.

\section{Acknowledgements}

The authors thank the patients for their willingness to cooperate with our study. This study was supported in part by grants from the Shanghai Science and Technology Committee (06DJ14004, 06DZ19504).

\section{References}

1. Cao AY, Hu Z and Shao ZM: Mutation screening of breast cancer susceptibility genes in Chinese high-risk families: the results will develop the genetic testing strategy in China. Breast Cancer Res Treat 120: 271-272, 2010.

2. Campeau PM, Foulkes WD and Tischkowitz MD: Hereditary breast cancer: new genetic developments, new therapeutic avenues. Hum Genet 124: 31-42, 2008.

3. Easton DF: Familial risks of breast cancer. Breast Cancer Res 4: 179-181, 2002.

4. Mohammed SN, Smith P, Hodgson SV, Fentiman IS, Miles DW, Barnes DM, Millis RR and Rubens RD: Family history and survival in premenopausal breast cancer. $\mathrm{Br} \mathrm{J}$ Cancer 77 : 2252-2256, 1998.

5. Verkooijen HM, Chappuis PO, Rapiti E, Vlastos G, Fioretta G, Sarp S, Sappino AP, Schubert H and Bouchardy C: Impact of familial risk factors on management and survival of early-onset breast cancer: a population-based study. Br J Cancer 94: 231-238, 2006.

6. Russo A, Herd-Smith A, Gestri D, Bianchi S, Vezzosi V, Rosselli Del Turco M and Cardona G: Does family history influence survival in breast cancer cases? Int J Cancer 99: 427-430, 2002.

7. Chun J, Pocock B, Joseph KA, El-Tamer M, Klein L and Schnabel F: Breast cancer risk factors in younger and older women. Ann Surg Oncol 16: 96-99, 2009.

8. Meiser B, Tucker K, Friedlander M, Barlow-Stewart K, Lobb E, Saunders C and Mitchell G: Genetic counselling and testing for inherited gene mutations in newly diagnosed patients with breast cancer: a review of the existing literature and a proposed research agenda. Breast Cancer Res 10: 216, 2008.
9. Nomizu T, Tsuchiya A, Kanno M, Katagata N, Watanabe F, Yamaki Y, Abe R and Miki Y: Clinicopathological features of hereditary breast cancer. Breast Cancer 4: 239-242, 1997.

10. Atchley DP, Albarracin CT, Lopez A, Valero V, Amos CI, Gonzalez-Angulo AM, Hortobagyi GN and Arun BK: Clinical and pathologic characteristics of patients with BRCA-positive and BRCA-negative breast cancer. J Clin Oncol 26: 4282-4288, 2008.

11. Hamann U and Sinn HP: Survival and tumor characteristics of German hereditary breast cancer patients. Breast Cancer Res Treat 59: 185-192, 2000

12. Moller P, Evans DG, Reis MM, Gregory H, Anderson E, Maehle L, Lalloo F, Howell A, Apold J, Clark N, Lucassen A and Steel CM: Surveillance for familial breast cancer: differences in outcome according to BRCA mutation status. Int J Cancer 121: 1017-1020, 2007.

13. Anders CK, Hsu DS, Broadwater G, Acharya CR, Foekens JA, Zhang Y, Wang Y, Marcom PK, Marks JR, Febbo PG, Nevins JR, Potti A and Blackwell KL: Young age at diagnosis correlates with worse prognosis and defines a subset of breast cancers with shared patterns of gene expression. J Clin Oncol 26: 3324-3330, 2008.

14. Han W, Kim SW, Park IA, Kang D, Kim SW, Youn YK, Oh SK, Choe KJ and Noh DY: Young age: an independent risk factor for disease-free survival in women with operable breast cancer. BMC Cancer 4: 82, 2004.

15. Livi L, Meattini I, Saieva C, Borghesi S, Scotti V, Petrucci A, Rampini A, Marrazzo L, Di Cataldo V, Bianchi S, Cataliotti L and Biti G: The impact of young age on breast cancer outcome. Eur J Surg Oncol 36: 639-645, 2010.

16. Maggard MA, O'Connell JB, Lane KE, Liu JH, Etzioni DA and Ko CY: Do young breast cancer patients have worse outcomes? J Surg Res 113: 109-113, 2003.

17. Jobsen JJ, Meerwaldt JH and van der Palen J: Family history in breast cancer is not a prognostic factor? Breast 9: 83-87, 2000.

18. Figueiredo JC, Ennis M, Knight JA, McLaughlin JR, Hood N, O'Malley F, Andrulis IL and Goodwin PJ: Influence of young age at diagnosis and family history of breast or ovarian cancer on breast cancer outcomes in a population-based cohort study. Breast Cancer Res Treat 105: 69-80, 2007.

19. Tang LC, Yin WJ, Di GH, Shen ZZ and Shao ZM: Unfavourable clinicopathologic features and low response rate to systemic adjuvant therapy: results with regard to poor survival in young Chinese breast cancer patients. Breast Cancer Res Treat 122: 95-104, 2010.

20. Dawood S, Broglio K, Buzdar AU, Hortobagyi GN and Giordano SH: Prognosis of women with metastatic breast cancer by HER 2 status and trastuzumab treatment: an institutionalbased review. J Clin Oncol 28: 92-98, 2010.

21. Murff HJ, Spigel DR and Syngal S: Does this patient have a family history of cancer? An evidence-based analysis of the accuracy of family cancer history. JAMA 292: 1480-1489, 2004.

22. Marcus JN, Watson P, Page DL, Narod SA, Lenoir GM, Tonin P, Linder-Stephenson L, Salerno G, Conway TA and Lynch HT: Hereditary breast cancer: pathobiology, prognosis, and BRCA1 and BRCA2 gene linkage. Cancer 77: 697-709, 1996.

23. Robson M, Gilewski T, Haas B, Levin D, Borgen P, Rajan P, Hirschaut Y, Pressman P, Rosen PP, Lesser ML, Norton L and Offit K: BRCA-associated breast cancer in young women. J Clin Oncol 16: 1642-1649, 1998

24. Slattery ML, Berry TD and Kerber RA: Is survival among women diagnosed with breast cancer influenced by family history of breast cancer? Epidemiology 4: 543-548, 1993.

25. Malone KE, Daling JR, Weiss NS, McKnight B, White E and Voigt LF: Family history and survival of young women with invasive breast carcinoma. Cancer 78: 1417-1425, 1996.

26. Rapiti E, Fioretta G, Verkooijen HM, Vlastos G, Schäfer P, Sappino AP, Kurtz J, Neyroud-Caspar I and Bouchardy C: Survival of young and older breast cancer patients in Geneva from 1990 to 2001. Eur J Cancer 41: 1446-1452, 2005.

27. Schouten LJ, Hupperets PS, Jager JJ, Volovics L, Wils JA, Verbeek AL and Blijham GH: Prognostic significance of etiological risk factors in early breast cancer. Breast Cancer Res Treat 43: 217-223, 1997. 